\title{
Fine-Scale Predictions of Distributions of Chagas Disease Vectors in the State of Guanajuato, Mexico
}

\author{
JORGE LÓPEZ-CÁRDENAS, ${ }^{1}$ FRANCISCO ERNESTO GONZALEZ BRAVO, ${ }^{2}$ \\ PAZ MARIA SALAZAR SCHETTINO ${ }^{3}$ JUAN CARLOS GALLAGA SOLORZANO, ${ }^{1}$ \\ ECTOR RAMÍREZ BARBA, ${ }^{4}$ JOEL MARTINEZ MENDEZ, ${ }^{4} \mathrm{~V}$. SÁNCHEZ-CORDERO,${ }^{5}$ \\ A. TOWNSEND PETERSON, ${ }^{6}$ AND J. M. RAMSEY ${ }^{7}$
}

J. Med. Entomol. 42(6): 1068-1081 (2005)

\begin{abstract}
One of the most daunting challenges for Chagas disease surveillance and control in Mexico is the lack of community level data on vector distributions. Although many states now have assembled representative domestic triatomine collections, only two triatomine specimens had been collected and reported previously from the state of Guanajuato. Field personnel from the state's Secretaría de Salud conducted health promotion activities in 43 of the 46 counties in the state and received donations of a total of 2,522 triatomine specimens between 1998 and 2002. All specimens were identified, and live insects examined for Trypanosoma cruzi. In an effort to develop fine-scale distributional data for Guanajuato, collection localities were georeferenced and ecological niches were modeled for each species by using evolutionary-computing approaches. Five species were collected: Triatoma mexicana (Herrich-Schaeffer), Triatoma longipennis (Usinger), Triatoma pallidipennis (Stål), Triatoma barberi (Usinger), and Triatoma dimidiata (Latreille) from 201 communities located at elevations of 870-2,200 m. Based on collection success, T. mexicana had the broadest dispersion, although niche mapping indicates that $T$. barberi represents the greatest risk for transmission of Chagas disease in the state. $T$. dimidiata was represented in collections by a single adult collected from one village outside the predicted area for all species. For humans, an estimated 3,755,380 individuals are at risk for vector transmission in the state, with an incidence of 3,500 new cases per year; overall seroprevalences of $2.6 \%$ indicate that 97,640 individuals are infected with $T$. cruzi at present, including 29,300 chronic cases.
\end{abstract}

KEY WORDS Chagas disease, Guanajuato, Mexico, triatomine, vector distribution

EighteEn tRiatomine (Triatominae: Reduviidae) species have been documented as vectors of Trypanosoma cruzi in Mexico (Zárate and Zárate 1985, Vidal-Acosta et al. 2000). Nine of these species are considered as primary vector species (Ramsey et al. 2003), and most belong to two species complexes (phyllosoma and protracta) in the genus Triatoma (Lent and Wygod-

${ }^{1}$ Guanajuato State Public Health Laboratory, Medical Entomology Laboratory Secretaria de Salud del Estado de Guanajuato. Calle Beta 204 Fracc. Delta, León, Guanajuato 37320, México.

2 Universidad de Guanajuato, Facultad de Enfermería y Obstetricia en León, Aquiles Serdán 914, col Obregón, León, Guanajuato 37320 , México.

${ }^{3}$ Medical School, Universidad Nacional Autónoma de México. Ciudad Universitaria, México D.F. 04510, México.

${ }^{4}$ Instituto de Salud Pública del Estado de Guanajuato, Tamazuca 4 Centro, Guanajuato, Guanajuato 36000, México.

${ }^{5}$ Instituto de Biología, Universidad Nacional Autónoma de México, Apartado Postal 70-153, México, D.F. 04510 México.

${ }^{6}$ Natural History Museum, The University of Kansas, Lawrence, KS 66045 .

${ }^{7}$ Centro de Investigaciones sobre Enfermedades Infecciosas (CISEI), Instituto Nacional de Salud Pública (INSP), Cuernavaca, Morelos, 62508 México. zinsky 1979). Multiple triatomine species are frequently found within a single region or state in Mexico. As a result, $>71 \%$ of the Mexican population is at direct risk of acquiring T. cruzi infections from triatomines, and $>96 \%$ of all $T$. cruzi transmission occurs via these vectors (Ramsey et al. 2003).

One of the most significant challenges for Chagas disease surveillance and control in Mexico is the lack of fine-scale (at the level of local communities) vector distribution data, as well as the almost complete ignorance of the disease-transmission characteristics and risk areas, both by the public health community and by at-risk populations. With improved malaria control in the country, in the last decade, "additional" operative vector control activities have focused primarily in urban environments owing to increases in classical and hemorrhagic dengue transmission. (To date, vector-borne disease control activities are budgeted only for malaria in the country.) Moreover, the recent decentralization of primary health care services to the state level has emphasized the need and the opportunity to review local and state health care priorities, and despite overburdened and shrinking 
Table 1. Communities where Triatoma spp. were collected in domestic habitats in the state of Gunajuato, $1998-2002$

\begin{tabular}{|c|c|c|c|c|c|c|c|}
\hline County & Code Cty. & Community & Code comm. & Longitude & Latitude & Altitude & Triatoma species \\
\hline Abasolo & 1 & La Colonia & 470 & 1013455 & 203400 & 1,690 & longipennis \\
\hline Abasolo & 1 & Abasolo & 1 & 1013145 & 202700 & 1,710 & pallidipennis \\
\hline Acambaro & 2 & Acambaro & 1 & 1004318 & 200153 & 1,860 & barberi \\
\hline Apaseo el Alto & 4 & Mandujano de Atepehuacan & 36 & 1003445 & 202555 & 1,940 & barberi \\
\hline Apaseo el Alto & 4 & Santa Cruz de Gamboa & 17 & 1003100 & 202318 & 2,010 & barberi \\
\hline Apaseo el Grande- & 5 & Ixtla tierra Blanca & 19 & 1003520 & 203911 & 1,890 & barberi \\
\hline Apaseo el Grande- & 5 & Tierra Blanca & 52 & 1003614 & 204141 & 2,070 & barberi \\
\hline Atarjea & 6 & Salitrillo & 37 & 0994510 & 211506 & 1,400 & mexicana \\
\hline Atarjea & 6 & Charcas & 7 & 0995138 & 211230 & 1,720 & mexicana \\
\hline Atarjea & 6 & El Chilarito & 9 & 0995130 & 211121 & 1,800 & mexicana \\
\hline Atarjea & 6 & Piedra Gorda & 17 & 0995208 & 211230 & 1,820 & mexicana \\
\hline Atarjea & 6 & La Joya & 11 & 0995152 & 211643 & 1,920 & mexicana \\
\hline Atarjea & 6 & Aldama & 3 & 0995006 & 211037 & 1,960 & mexicana \\
\hline Atarjea & 6 & Villa Aldama & 3 & 0995006 & 211037 & 1,960 & mexicana \\
\hline Atarjea & 6 & El Apartadero & 26 & 0995019 & 211247 & 1,980 & mexicana \\
\hline Atarjea & 6 & la tapona & 21 & 0995220 & 211100 & 2,020 & mexicana \\
\hline Atarjea & 6 & Hacienda de Charcas & 7 & 0995138 & 211230 & 1,720 & mexicana \\
\hline Celaya & 7 & Santa Teresa & 177 & 1005126 & 203814 & 1,780 & barberi \\
\hline Celaya & 7 & Rincón de Tamayo & 143 & 1004520 & 202518 & 1,790 & barberi \\
\hline Comonfort & 9 & Empalme Escobedo & 10 & 1004500 & 204017 & 1,790 & barberi \\
\hline Comonfort & 9 & Xoconoxtle & 15 & 1005702 & 204208 & 1,850 & barberi \\
\hline Comonfort & 9 & Pocitos de San Antonio & 34 & 1005634 & 204308 & 1,900 & barberi \\
\hline Comonfort & 9 & Potrero & 35 & 1003848 & 204530 & 1,910 & barberi \\
\hline Comonfort & 9 & Landín & 18 & 1005422 & 204515 & 1,960 & barberi \\
\hline Comonfort & 9 & San Antonio de Corrales & 41 & 1005750 & 204440 & 2,000 & barberi \\
\hline Coroneo & 10 & Coroneo & 1 & 1002153 & 201151 & 2,270 & barberi \\
\hline Cuer $1 / 3$ maro & 12 & San Gregorio & 26 & 1013740 & 203245 & 1,700 & longipennis \\
\hline Cuer $1 / 3$ maro & 12 & San Gregorio & 26 & 1013740 & 203245 & 1,700 & pallidipennis \\
\hline Dolores Hidalgo & 14 & Dolores Hidalgo & 1 & 1005558 & 210918 & 1,920 & barberi \\
\hline Dolores Hidalgo & 14 & Cieneguilla & 57 & 1005615 & 210423 & 1,950 & barberi \\
\hline Dolores Hidalgo & 14 & La Trinidad & 351 & 1005924 & 210851 & 1,970 & barberi \\
\hline Dolores Hidalgo & 14 & La Joya & 140 & 1004226 & 210707 & 1,980 & barberi \\
\hline Dolores Hidalgo & 14 & Ojo Zarco & 186 & 1005155 & 210143 & 1,980 & barberi \\
\hline Dolores Hidalgo & 14 & San Isidro Durazno & 400 & 1005646 & 210336 & 1,980 & barberi \\
\hline Dolores Hidalgo & 14 & Santiaguillo & 315 & 1005859 & 210052 & 1,980 & barberi \\
\hline Dolores Hidalgo & 14 & Palacio de Abajo & 191 & 1005912 & 210120 & 2,000 & barberi \\
\hline Dolores Hidalgo & 14 & Río Azul & 254 & 1010210 & 210815 & 2,000 & barberi \\
\hline Dolores Hidalgo & 14 & Los Ju11/3rez & 141 & 1010138 & 211119 & 2,030 & barberi \\
\hline Dolores Hidalgo & 14 & San Isidro el Sisote & 285 & 1010117 & 210506 & 2,040 & barberi \\
\hline Dolores Hidalgo & 14 & Los Quijotes & 236 & 1010415 & 211004 & 2,050 & barberi \\
\hline Dolores Hidalgo & 14 & Terrero de Trancas & 343 & 1010242 & 211037 & 2,050 & barberi \\
\hline Dolores Hidalgo & 14 & Pereas de Arriba & 192 & 1010152 & 210100 & 2,060 & barberi \\
\hline Dolores Hidalgo & 14 & Santa B1/3rbara & 306 & 1010035 & 210254 & 2,060 & barberi \\
\hline Dolores Hidalgo & 14 & Refugio de Trancas & 249 & 1010410 & 211121 & 2,070 & barberi \\
\hline Dolores Hidalgo & 14 & Silleros & 322 & 1010037 & 210420 & 2,120 & barberi \\
\hline Dolores Hidalgo & 14 & Tequisquiapan & 340 & 1004912 & 210447 & 1,970 & mexicana \\
\hline Huanímaro & 16 & La Tinaja & 26 & 1012830 & 202320 & 1,790 & pallidipennis \\
\hline Huanímaro & 16 & La Tinaja & 26 & 1012830 & 202320 & 1,790 & longipennis \\
\hline Irapuato & 17 & la caja & 61 & 1012615 & 204240 & 1,710 & barberi \\
\hline Irapuato & 17 & san josé de Cano & 148 & 1012406 & 203820 & 1,720 & barberi \\
\hline Irapuato & 17 & Irapuato & 1 & 1012052 & 204023 & 1,730 & barberi \\
\hline Irapuato & 17 & Serrano & 167 & 1012343 & 204640 & 1,740 & barberi \\
\hline Irapuato & 17 & La Caja & 61 & 1012615 & 204240 & 1,710 & longipennis \\
\hline Irapuato & 17 & El Coecillo & 72 & 1012426 & 203757 & 1,720 & longipennis \\
\hline Irapuato & 17 & Gpe. De Rivera & 93 & 1012808 & 203621 & 1,720 & longipennis \\
\hline Irapuato & 17 & san josé de Cano & 148 & 1012406 & 203820 & 1,720 & longipennis \\
\hline Irapuato & 17 & Irapuato & 1 & 1012052 & 204023 & 1,730 & longipennis \\
\hline Irapuato & 17 & San Luis Janamo & 761 & 1012615 & 203407 & 1,730 & pallidipennis \\
\hline Juventino Rosas & 35 & Juventino & 1 & 1005943 & 203832 & 1,750 & barberi \\
\hline Juventino Rosas & 35 & mesa de acosta & 33 & 1010433 & 204453 & 1,970 & barberi \\
\hline Juventino Rosas & 35 & La Tinaja & 80 & 1010218 & 204215 & 2,000 & barberi \\
\hline Juventino Rosas & 35 & San José del Sauz & 63 & 1005901 & 204027 & 1,800 & barberi \\
\hline Jaral del Progreso & 18 & Cerrito de Camargo & 4 & 1010233 & 202505 & 1,720 & barberi \\
\hline León & 20 & Ejido de los López & 1121 & 1013448 & 210224 & 1,800 & longipennis \\
\hline León & 20 & Plan De Ayala & 401 & 1014316 & 210421 & 1,770 & mexicana \\
\hline León & 20 & Ejido de los López & 1121 & 1013448 & 210224 & 1,800 & mexicana \\
\hline León & 20 & La Cantera & 1103 & 1013204 & 210436 & 1,860 & mexicana \\
\hline Manuel Doblado & 8 & Ciudad M. Doblado & 1 & 1015658 & 204338 & 1,720 & barberi \\
\hline Manuel Doblado & 8 & Atotonilquillo & 4 & 1015425 & 203835 & 1,730 & barberi \\
\hline Manuel Doblado & 8 & El Tecu $1 \frac{1}{3 n}$ & 146 & 1015152 & 204935 & 1,730 & barberi \\
\hline Manuel Doblado & 8 & Rancho Nuevo Atotonilquillo & 95 & 1015345 & 204030 & 1,740 & barberi \\
\hline Manuel Doblado & 8 & Cerrito de santiago & 22 & 1015509 & 203854 & 1,740 & barberi \\
\hline
\end{tabular}


Table 1. (Continued)

\begin{tabular}{|c|c|c|c|c|c|c|c|}
\hline County & Code Cty. & Community & Code comm. & Longitude & Latitude & Altitude & Triatoma species \\
\hline Manuel Doblado & 8 & Frias & 39 & 1015950 & 204750 & 1,740 & barberi \\
\hline Manuel Doblado & 8 & Zapote de Adjuntas & 166 & 1015100 & 204007 & 1,750 & barberi \\
\hline Manuel Doblado & 8 & capulin chico & 17 & 1014700 & 204010 & 1,760 & barberi \\
\hline Manuel Doblado & 8 & San Juan de la Puerta & 124 & 1014245 & 204345 & 1,710 & longipennis \\
\hline Manuel Doblado & 8 & Ciudad M. Doblado & 1 & 1015658 & 204338 & 1,720 & longipennis \\
\hline Manuel Doblado & 8 & Atotonilquillo & 4 & 1015425 & 203835 & 1,730 & longipennis \\
\hline Manuel Doblado & 8 & Camargo de Adjuntas & 2 & 1015035 & 204025 & 1,730 & longipennis \\
\hline Manuel Doblado & 8 & El Tecu $1 / 3 n$ & 146 & 1015152 & 204935 & 1,730 & longipennis \\
\hline Manuel Doblado & 8 & Cerrito de santiago & 22 & 1015509 & 203854 & 1,740 & longipennis \\
\hline Manuel Doblado & 8 & Carrizo de Godinez & 19 & 1014945 & 204045 & 1,750 & longipennis \\
\hline Manuel Doblado & 8 & Zapote de Adjuntas & 166 & 1015100 & 204007 & 1,750 & longipennis \\
\hline Manuel Doblado & 8 & El Varal & 468 & 1014828 & 204948 & 1,800 & longipennis \\
\hline Manuel Doblado & 8 & Piedra Parada & 75 & 1014827 & 204445 & 1,810 & longipennis \\
\hline Manuel Doblado & 8 & La Soledad de Leones & 141 & 1015521 & 203608 & 1,880 & longipennis \\
\hline Manuel Doblado & 8 & San Antonio de la presa & 110 & 1015653 & 203830 & 1,790 & pallidipennis \\
\hline Manuel Doblado & 8 & Cerrito de santiago & 22 & 1015509 & 203854 & 1,740 & pallidipennis \\
\hline Manuel Doblado & 8 & El Varal & 468 & 1014828 & 204948 & 1,800 & pallidipennis \\
\hline Manuel Doblado & 8 & Frias & 39 & 1015950 & 204750 & 1,740 & pallidipennis \\
\hline Manuel Doblado & 8 & San Pablo & 130 & 1014922 & 204503 & 1,730 & pallidipennis \\
\hline Manuel Doblado & 8 & Vado de Zapién & 162 & 1014905 & 204052 & 1,730 & pallidipennis \\
\hline Manuel Doblado & 8 & Zapote de Adjuntas & 166 & 1015100 & 204007 & 1,750 & pallidipennis \\
\hline Moroleón & 21 & El Salto & 18 & 1011100 & 200256 & 1,970 & barberi \\
\hline Moroleón & 21 & Moroleón & 1 & 1011125 & 200743 & 1,810 & barberi \\
\hline Pénjamo & 23 & Sauz de Méndez & 305 & 1015718 & 202910 & 1,700 & barberi \\
\hline Pénjamo & 23 & Pénjamo & 1 & 1014321 & 202548 & 1,770 & barberi \\
\hline Pénjamo & 23 & El Sauz & 306 & 1015503 & 202853 & 1,800 & barberi \\
\hline Pénjamo & 23 & Huerta Grande & 423 & 1015353 & 202837 & 1,850 & barberi \\
\hline Pénjamo & 23 & Mezquite de Luna & 177 & 1015334 & 201747 & 1,690 & longipennis \\
\hline Pénjamo & 23 & Ordeña de Barajas & 194 & 1014028 & 202050 & 1,700 & longipennis \\
\hline Pénjamo & 23 & Purísima & 235 & 1014555 & 201238 & 1,710 & longipennis \\
\hline Pénjamo & 23 & Pénjamo & 1 & 1014321 & 202548 & 1,770 & longipennis \\
\hline Pénjamo & 23 & Magallanes & 167 & 1014150 & 202746 & 1,820 & longipennis \\
\hline Pénjamo & 23 & Zapote de Cestao & 344 & 1014035 & 202900 & 1,820 & longipennis \\
\hline Pueblo Nuevo & 24 & Pueblo Nuevo & 1 & 1012225 & 203133 & 1,700 & longipennis \\
\hline Pueblo Nuevo & 24 & Villa de Guadalupe & 15 & 1012256 & 203356 & 1,720 & pallidipennis \\
\hline Romita & 26 & Santa Rosa de Rivas & 74 & 1013211 & 204653 & 1,740 & longipennis \\
\hline Salamanca & 27 & La Capilla & 42 & 1011800 & 203320 & 1,700 & barberi \\
\hline Salamanca & 27 & La Ordeña & 99 & 1010706 & 204122 & 1,810 & barberi \\
\hline Salamanca & 27 & El Estanco & 67 & 1010945 & 204519 & 1,830 & barberi \\
\hline Salamanca & 27 & La Joyita de Villafaña & 79 & 1010818 & 204740 & 1,990 & barberi \\
\hline San Felipe & 30 & Peñuelas & 163 & 1011505 & 211725 & 2130 & barberi \\
\hline San Felipe & 30 & El Aro & 13 & 1012151 & 211000 & 2,150 & barberi \\
\hline San Felipe & 30 & San Vicente & 254 & 1012029 & 211404 & 2,150 & barberi \\
\hline San Luis de la Paz & 33 & Las Teresas & 223 & 1001708 & 213510 & 1,400 & barberi \\
\hline San Luis de la Paz & 33 & El Realito & 165 & 1001330 & 213613 & 1,060 & mexicana \\
\hline San Luis de la Paz & 33 & El Guamúchil & 69 & 1001524 & 213711 & 1,100 & mexicana \\
\hline San Luis de la Paz & 33 & Paso de Mezquites & 512 & 1001420 & 213616 & 1,200 & mexicana \\
\hline San Luis de la Paz & 33 & Huizaches & 73 & 1001715 & 213424 & 1,260 & mexicana \\
\hline San Luis de la Paz & 33 & El higueron & 72 & 1002224 & 214015 & 1,300 & mexicana \\
\hline San Luis de la Paz & 33 & El Bramador & 415 & 1001808 & 213608 & 1,340 & mexicana \\
\hline San Luis de la Paz & 33 & El Capadero & 249 & 1001605 & 213603 & 1,400 & mexicana \\
\hline San Luis de la Paz & 33 & Las Teresas & 223 & 1001708 & 213510 & 1,400 & mexicana \\
\hline San Luis de la Paz & 33 & Ojo de Agua & 364 & 1001713 & 213453 & 1,400 & mexicana \\
\hline San Luis de la Paz & 33 & Mesa de la Estacada & 102 & 1001820 & 213350 & 1,460 & mexicana \\
\hline San Luis de la Paz & 33 & El Saucillo & 323 & 1001658 & 213654 & 1,480 & mexicana \\
\hline San Luis de la Paz & 33 & El Aguacate & 484 & 1001958 & 213955 & 1,500 & mexicana \\
\hline San Luis de la Paz & 33 & Mesita de Camaron & 293 & 1001950 & 214031 & 1,540 & mexicana \\
\hline San Luis de la Paz & 33 & Cañada del Potrero & 19 & 1002429 & 213949 & 1,600 & mexicana \\
\hline San Luis de la Paz & 33 & Rincón del Pitayo & 775 & 1001531 & 213103 & 1,620 & mexicana \\
\hline San Luis de la Paz & 33 & La Soledad & 218 & 1004015 & 211110 & 1,980 & mexicana \\
\hline San Luis de la Paz & 33 & Las Adjuntas & 2 & 1003223 & 212745 & 2,050 & mexicana \\
\hline San Luis de la Paz & 33 & El potrero del Sauz & 325 & 1002547 & 212018 & 2,100 & mexicana \\
\hline San Luis de la Paz & 33 & San Luis de la Paz & 1 & 1003100 & 211751 & 2,100 & mexicana \\
\hline San Luis de la Paz & 33 & Ejido de Ortega & 119 & 1002416 & 211625 & 2,200 & mexicana \\
\hline San Miguel Allende & 3 & Banda & 18 & 1004919 & 205750 & 1,850 & mexicana \\
\hline San Miguel Allende & 3 & Cienega de Juana Ruiz & 64 & 1005203 & 205410 & 1,860 & mexicana \\
\hline San Miguel Allende & 3 & Corral de Piedras & 77 & 1003536 & 205835 & 2,060 & mexicana \\
\hline San Miguel Allende & 3 & El Kiwi & 512 & 1003907 & 205302 & 2,060 & mexicana \\
\hline Santa Catarina & 34 & Llano Blanco & 19 & 1000328 & 210826 & 1,560 & barberi \\
\hline Santa Catarina & 34 & El Zapote & 47 & 1000602 & 210954 & 1,600 & dimidiata \\
\hline Santa Catarina & 34 & Cruz de Diego & 11 & 1000207 & 210759 & 1,540 & mexicana \\
\hline Santa Catarina & 34 & Ortega & 21 & 1000116 & 210744 & 1,540 & mexicana \\
\hline Santa Catarina & 34 & Tres Alamos & 34 & 1000201 & 210816 & 1,550 & mexicana \\
\hline
\end{tabular}


Table 1. (Continued)

\begin{tabular}{|c|c|c|c|c|c|c|c|}
\hline County & Code Cty. & Community & Code comm. & Longitude & Latitude & Altitude & Triatoma species \\
\hline Santa Catarina & 34 & Las Limitas & 18 & 1000308 & 210818 & 1,560 & mexicana \\
\hline Santa Catarina & 34 & Llano Blanco & 19 & 1000328 & 210826 & 1,560 & mexicana \\
\hline Santa Catarina & 34 & Santa Catarina & 1 & 1000406 & 210820 & 1,560 & mexicana \\
\hline Santa Catarina & 34 & Agua Buena & 2 & 1000401 & 210844 & 1,570 & mexicana \\
\hline Santa Catarina & 34 & El Tablón & 32 & 1000441 & 210803 & 1,570 & mexicana \\
\hline Santa Catarina & 34 & Barrio de Santa Cruz & 27 & 1000447 & 210748 & 1,580 & mexicana \\
\hline Santa Catarina & 34 & Peña Colorada & 23 & 1000431 & 210904 & 1,590 & mexicana \\
\hline Santa Catarina & 34 & Charco del Muerto & 13 & 1000607 & 210748 & 1,600 & mexicana \\
\hline Santa Catarina & 34 & El Mezquital & 41 & 1000341 & 210757 & 1,600 & mexicana \\
\hline Santa Catarina & 34 & El Zapote & 47 & 1000602 & 210954 & 1,600 & mexicana \\
\hline Santa Catarina & 34 & La Cantera & 6 & 1000524 & 210705 & 1,630 & mexicana \\
\hline Santa Catarina & 34 & Corral Blanco & 9 & 1000632 & 211020 & 1,640 & mexicana \\
\hline Santa Catarina & 34 & La Rusia & 24 & 1000428 & 210613 & 1,670 & mexicana \\
\hline Santa Catarina & 34 & El Chapin & 12 & 1000359 & 210532 & 1,700 & mexicana \\
\hline Santa Catarina & 34 & San Jose del Chilar & 26 & 1000221 & 210951 & 1,800 & mexicana \\
\hline Santa Catarina & 34 & San José del Chilar & 26 & 1000221 & 210951 & 1,800 & mexicana \\
\hline Santa Catarina & 34 & El Nogal & 20 & 1000548 & 211157 & 1,850 & mexicana \\
\hline Santa Catarina & 34 & Paredes & 22 & 1000528 & 211252 & 2,150 & mexicana \\
\hline Santa Catarina & 34 & La Faja & 44 & 1000400 & 210803 & 1,570 & mexicana \\
\hline Santa Catarina & 34 & Los Juan Diegos & 16 & 1000318 & 210812 & 1,550 & mexicana \\
\hline Silao & 37 & Silao & 1 & 1012537 & 205633 & 1,780 & barberi \\
\hline Silao & 37 & Bajío de Bonillas & 6 & 1012944 & 205654 & 1,790 & barberi \\
\hline Silao & 37 & Chichimequillas & 31 & 1012634 & 210215 & 1,850 & barberi \\
\hline Sn. Fco. Del Rincón & 31 & El Refugio & 113 & 1014448 & 205010 & 1,850 & longipennis \\
\hline Sn. Fco. Del Rincón & 31 & El Refugio & 113 & 1014448 & 205010 & 1,850 & pallidipennis \\
\hline Tarimoro & 39 & Tarimoro & 1 & 1004530 & 201715 & 1,770 & barberi \\
\hline Tarimoro & 39 & Huapango & 19 & 1004105 & 202150 & 2,150 & barberi \\
\hline Tierra Blanca & 40 & Monte Prieto & 28 & 1000810 & 210635 & 1,680 & barberi \\
\hline Tierra Blanca & 40 & Las Moras & 29 & 1000948 & 210423 & 1,750 & barberi \\
\hline Tierra Blanca & 40 & El Guadalupe & 18 & 1001103 & 210332 & 1,850 & barberi \\
\hline Tierra Blanca & 40 & Cano de San Isidro & 57 & 1001335 & 210350 & 1,870 & barberi \\
\hline Tierra Blanca & 40 & Cerro Colorado & 9 & 1001355 & 210610 & 1,890 & barberi \\
\hline Tierra Blanca & 40 & Cuesta de Peñones & 11 & 1001358 & 210502 & 1,900 & barberi \\
\hline Tierra Blanca & 40 & Milpa Blanca & 27 & 1000826 & 210453 & 1,900 & barberi \\
\hline Tierra Blanca & 40 & Rincón del Cano & 38 & 1001429 & 210323 & 1,900 & barberi \\
\hline Tierra Blanca & 40 & Peña Blanca & 33 & 1000802 & 210752 & 1,640 & mexicana \\
\hline Tierra Blanca & 40 & El Tepetate & 44 & 1000921 & 210651 & 1,680 & mexicana \\
\hline Tierra Blanca & 40 & Fracción de Guadalupe & 16 & 1000911 & 210738 & 1,680 & mexicana \\
\hline Tierra Blanca & 40 & Monte Prieto & 28 & 1000810 & 210635 & 1,680 & mexicana \\
\hline Tierra Blanca & 40 & Don Blas & 13 & 1000914 & 210625 & 1,700 & mexicana \\
\hline Tierra Blanca & 40 & Tierra Blanca & 1 & 1000929 & 210600 & 1,700 & mexicana \\
\hline Tierra Blanca & 40 & El Mezquite & 25 & 1000749 & 210601 & 1,730 & mexicana \\
\hline Tierra Blanca & 40 & Villa Unión & 63 & 1000941 & 210441 & 1,740 & mexicana \\
\hline Tierra Blanca & 40 & Cieneguilla & 10 & 1001101 & 210448 & 1,750 & mexicana \\
\hline Tierra Blanca & 40 & Las Moras & 29 & 1000948 & 210423 & 1,750 & mexicana \\
\hline Tierra Blanca & 40 & El Salto & 40 & 1000912 & 210327 & 1,760 & mexicana \\
\hline Tierra Blanca & 40 & El Zapote & 64 & 1000942 & 210510 & 1,760 & mexicana \\
\hline Tierra Blanca & 40 & Torrecitas & 47 & 1001200 & 210454 & 1,770 & mexicana \\
\hline Tierra Blanca & 40 & Juanica & 56 & 1001042 & 210414 & 1,780 & mexicana \\
\hline Tierra Blanca & 40 & El Picacho & 35 & 1001124 & 210421 & 1,800 & mexicana \\
\hline Tierra Blanca & 40 & El Progreso & 87 & 1001108 & 210432 & 1,800 & mexicana \\
\hline Tierra Blanca & 40 & El Guadalupe & 18 & 1001103 & 210332 & 1,850 & mexicana \\
\hline Tierra Blanca & 40 & El Sauz & 42 & 1000840 & 210214 & 1,850 & mexicana \\
\hline Tierra Blanca & 40 & fraccón del Cano & 6 & 1001349 & 210402 & 1,850 & mexicana \\
\hline Tierra Blanca & 40 & El Sauz del Salto & 42 & 1000840 & 210214 & 1,850 & mexicana \\
\hline Tierra Blanca & 40 & Las Adjuntas & 2 & 1001043 & 210212 & 1,860 & mexicana \\
\hline Tierra Blanca & 40 & Cano de San Isidro & 57 & 1001335 & 210350 & 1,870 & mexicana \\
\hline Tierra Blanca & 40 & Cerro Colorado & 9 & 1001355 & 210610 & 1,890 & mexicana \\
\hline Tierra Blanca & 40 & Cuesta de Peñones & 11 & 1001358 & 210502 & 1,900 & mexicana \\
\hline Tierra Blanca & 40 & Milpa Blanca & 27 & 1000826 & 210453 & 1,900 & mexicana \\
\hline Tierra Blanca & 40 & Rincón del Cano & 38 & 1001429 & 210323 & 1,900 & mexicana \\
\hline Tierra Blanca & 40 & La Estancia & 15 & 1000829 & 210046 & 1,950 & mexicana \\
\hline Tierra Blanca & 40 & La Mesita & 24 & 1000858 & 205924 & 2,090 & mexicana \\
\hline Tierra Blanca & 40 & El Apartadero & 53 & 1000819 & 205918 & 2,160 & mexicana \\
\hline Tierra Blanca & 40 & Peña blanca dos & 34 & 1001057 & 210508 & 1,750 & mexicana \\
\hline Tierra Blanca & 40 & El Sauz del Salto & 42 & 1000840 & 210214 & 1,850 & mexicana \\
\hline Tierra Blanca & 40 & Rincon del Cano & 38 & 1001429 & 210323 & 1,900 & mexicana \\
\hline Tierra Blanca & 40 & Cerro colorado & 9 & 1001355 & 210610 & 1,890 & mexicana \\
\hline Uriangato & 41 & El Charco & 10 & 1010737 & 200846 & 1,950 & barberi \\
\hline Uriangato & 41 & El Cerro & 5 & 1011211 & 200223 & 2,060 & barberi \\
\hline Uriangato & 41 & Lagunilla del Rico & 16 & 1010524 & 200531 & 2,120 & barberi \\
\hline Valle de Santiago & 42 & La Barquilla & 5 & 1012625 & 202005 & 1,700 & barberi \\
\hline Valle de Santiago & 42 & Salitre de Adjuntas & 90 & 1012635 & 201905 & 1,710 & barberi \\
\hline
\end{tabular}


Table 1. (Continued)

\begin{tabular}{|c|c|c|c|c|c|c|c|}
\hline County & Code Cty. & Community & Code comm. & Longitude & Latitude & Altitude & Triatoma species \\
\hline Valle de Santiago & 42 & Valle de Santiago & 1 & 1011130 & 202330 & 1,720 & barberi \\
\hline Valle de Santiago & 42 & Potrerillo de Torres & 69 & 1011042 & 201538 & 1,730 & barberi \\
\hline Valle de Santiago & 42 & Las Jícamas & 43 & 1012130 & 201638 & 1,850 & barberi \\
\hline Valle de Santiago & 42 & Mesa de san Agustín & 54 & 1011725 & 202014 & 1,850 & barberi \\
\hline Valle de Santiago & 42 & Cerro Blanco & 14 & 1011802 & 201825 & 2,060 & barberi \\
\hline Valle de Santiago & 42 & $\begin{array}{l}\text { Armadillo } \\
\text { and }\end{array}$ & 4 & 1011333 & 201700 & 2,080 & barberi \\
\hline Valle de Santiago & 42 & La Barquilla & 5 & 1012625 & 202005 & 1,700 & longipennis \\
\hline Victoria & 43 & Calera & 12 & 1001708 & 211209 & 1,950 & mexicana \\
\hline Victoria & 43 & Cerrito Colorado & 105 & 1001020 & 211323 & 1,770 & barberi \\
\hline Victoria & 43 & El Refugio & 71 & 1001820 & 211245 & 1,900 & barberi \\
\hline Victoria & 43 & Milpillas & 46 & 1001434 & 212040 & 2,140 & barberi \\
\hline Victoria & 43 & Los Tres pasos & & 1000957 & 213108 & 1,020 & mexicana \\
\hline Victoria & 43 & Agua Fría & 3 & 1001044 & 213046 & 1,040 & mexicana \\
\hline Victoria & 43 & El Tepehuaje & 85 & 1000843 & 213144 & 1,060 & mexicana \\
\hline Victoria & 43 & Terrón Blanco & 87 & 1001228 & 213732 & 1,100 & mexicana \\
\hline Victoria & 43 & Alamos de Mtz. & 7 & 1001024 & 213606 & 1,100 & mexicana \\
\hline Victoria & 43 & San Antonio de la Cueva & 78 & 1001247 & 212620 & 1,200 & mexicana \\
\hline Victoria & 43 & Buenavista de Guadalupe & 11 & 1000550 & 213408 & 1,300 & mexicana \\
\hline Victoria & 43 & Cieneguilla & 108 & 1001628 & 211451 & 1,340 & mexicana \\
\hline Victoria & 43 & Potrerillos & 64 & 1001131 & 212554 & 1,400 & mexicana \\
\hline Victoria & 43 & Los Colorados & 140 & 1000702 & 212907 & 1,420 & mexicana \\
\hline Victoria & 43 & El Chilcuague & 24 & 1001149 & 212402 & 1,560 & mexicana \\
\hline Victoria & 43 & El Quelite & 69 & 1000921 & 211120 & 1,700 & mexicana \\
\hline Victoria & 43 & La Salitrera & 76 & 1000841 & 211104 & 1,700 & mexicana \\
\hline Victoria & 43 & Las Negritas & 150 & 1001655 & 212400 & 1,700 & mexicana \\
\hline Victoria & 43 & Milpillas de Santiago & 45 & 1001044 & 211159 & 1,720 & mexicana \\
\hline Victoria & 43 & Victoria & 1 & 1001253 & 211240 & 1,740 & mexicana \\
\hline Victoria & 43 & El Carrizal de Higueras & 215 & 1001242 & 211153 & 1,750 & mexicana \\
\hline Victoria & 43 & El Tepetate & 179 & 1001045 & 211245 & 1,750 & mexicana \\
\hline Victoria & 43 & Las Higueras & 215 & 1001242 & 211153 & 1,750 & mexicana \\
\hline Victoria & 43 & El Tasajillo & 84 & 1001131 & 211238 & 1,750 & mexicana \\
\hline Victoria & 43 & Cerrito Colorado & 105 & 1001020 & 211323 & 1,770 & mexicana \\
\hline Victoria & 43 & Cerro Grande & 72 & 1001156 & 211100 & 1,770 & mexicana \\
\hline Victoria & 43 & Los Remedios & 72 & 1001156 & 211100 & 1,770 & mexicana \\
\hline Victoria & 43 & Rancho Viejo & 72 & 1001156 & 211100 & 1,770 & mexicana \\
\hline Victoria & 43 & Jacalasuchil & 95 & 1001240 & 211126 & 1,790 & mexicana \\
\hline Victoria & 43 & Misión de Arnedo & 48 & 1001505 & 211348 & 1,790 & mexicana \\
\hline Victoria & 43 & Corralillos & 32 & 1001149 & 211009 & 1,820 & mexicana \\
\hline Victoria & 43 & Corralillos & 22 & 1001149 & 211009 & 1,820 & mexicana \\
\hline Victoria & 43 & La Simona & 82 & 1001548 & 211615 & 1,860 & mexicana \\
\hline Victoria & 43 & Corral de Piedras & 21 & 1001431 & 211221 & 1,870 & mexicana \\
\hline Victoria & 43 & Hda. De Higueras & 304 & 1001022 & 211525 & 1,880 & mexicana \\
\hline Victoria & 43 & Negritas & 53 & 1001758 & 211327 & 1,890 & mexicana \\
\hline Victoria & 43 & El Refugio & 71 & 1001820 & 211245 & 1,900 & mexicana \\
\hline Victoria & 43 & Sombrerete & 83 & 1001654 & 211622 & 1,900 & mexicana \\
\hline Victoria & 43 & Panales & 58 & 1001501 & 211535 & 1,920 & mexicana \\
\hline victoria & 43 & La Estancia & 28 & 1001340 & 211546 & 1,940 & mexicana \\
\hline Victoria & 43 & El Carrizo & 17 & 1000928 & 211438 & 1,950 & mexicana \\
\hline Victoria & 43 & Loma de los Chilitos & 35 & 1001834 & 211741 & 1,960 & mexicana \\
\hline Victoria & 43 & Nogal Chico & 54 & 1001544 & 211737 & 1,960 & mexicana \\
\hline Victoria & 43 & El Sauz de Arriba & 80 & 1001740 & 211750 & 2,000 & mexicana \\
\hline Victoria & 43 & La Gavia Chica & 29 & 1001824 & 211757 & 2,000 & mexicana \\
\hline Victoria & 43 & Capilla Blanca & 15 & 1001952 & 211307 & 2,010 & mexicana \\
\hline Victoria & 43 & Milpillas & 46 & 1001434 & 212040 & 2,140 & mexicana \\
\hline Victoria & 43 & Los Remedios & 72 & 1001156 & 211100 & 1,770 & barberi \\
\hline Xichú & 45 & Ojo de Agua & 62 & 0994746 & 212652 & 1,400 & mexicana \\
\hline Xichú & 45 & Los Cocos & 68 & 0994917 & 212458 & 900 & mexicana \\
\hline Xichú & 45 & El Platanal & 42 & 0995124 & 212615 & 1,020 & mexicana \\
\hline Xichú & 45 & La Pila & 39 & 0995949 & 212641 & 1,100 & mexicana \\
\hline Xichú & 45 & Piedras Pintadas & 38 & 0995552 & 211600 & 1,160 & mexicana \\
\hline Xichú & 45 & Palomas & 36 & 0995105 & 212238 & 1,240 & mexicana \\
\hline Xichú & 45 & Paso de Guillermo & 37 & 995530 & 211317 & 1,380 & mexicana \\
\hline Xichú & 45 & Llano Grande & 22 & 1000440 & 211521 & 2,040 & mexicana \\
\hline Xichú & 45 & Piedras Pintadas & 38 & 0995552 & 211600 & 1,160 & barberi \\
\hline Xichú & 45 & Paso de Guillermo & 37 & 995530 & 211317 & 1,380 & barberi \\
\hline Xichú & 45 & Noria del Maltrato & 31 & 995629 & 212120 & 880 & mexicana \\
\hline Xichú & 45 & Llanetes & 21 & 995739 & 212058 & 900 & mexicana \\
\hline Xichú & 45 & La Majada & 69 & 994920 & 212534 & 950 & mexicana \\
\hline Xichú & 45 & La Escondida & 67 & 0995950 & 213105 & 1,070 & mexicana \\
\hline Xichú & 45 & San Diego de los Pitayos & 49 & 995752 & 211655 & 1,080 & mexicana \\
\hline Xichú & 45 & Adjuntas & 2 & 1000135 & 212025 & 1,100 & mexicana \\
\hline
\end{tabular}


Table 1. (Continued)

\begin{tabular}{|c|c|c|c|c|c|c|c|}
\hline County & Code Cty. & Community & Code comm. & Longitude & Latitude & Altitude & Triatoma species \\
\hline Xichú & 45 & La Salitrera & 48 & 0995503 & 211622 & 1,200 & mexicana \\
\hline Xichú & 45 & Los Potrerillos & 77 & 0995838 & 211622 & 1,200 & mexicana \\
\hline Xichú & 45 & El Roblar & 64 & 0994813 & 212625 & 1,300 & mexicana \\
\hline Xichú & 45 & Xichú & 1 & 1000320 & 211755 & 1,300 & mexicana \\
\hline Xichú & 45 & La Cieneguita & 13 & 0995708 & 211433 & 1,340 & mexicana \\
\hline Xichú & 45 & Mision de Sta. Rosa & 30 & 1000511 & 211713 & 1,560 & mexicana \\
\hline Xichú & 45 & Agua Zarca & 5 & 0995520 & 211758 & 1,600 & mexicana \\
\hline Yuriria & 46 & San Pablo Casacuar1/3n & 79 & 1010230 & 201200 & 1,740 & barberi \\
\hline Yuriria & 46 & San José Otonguitiro & 71 & 1011458 & 201131 & 1,740 & barberi \\
\hline Yuriria & 46 & Juan Lucas & 27 & 1011823 & 201051 & 1,740 & barberi \\
\hline Yuriria & 46 & Yuriria & 1 & 1010740 & 201240 & 1,740 & barberi \\
\hline Yuriria & 46 & San Andrés Calera & 61 & 1012300 & 200932 & 1,860 & barberi \\
\hline Yuriria & 46 & Tejocote de Calera & 86 & 1012346 & 201048 & 1,900 & barberi \\
\hline Yuriria & 46 & Tinaja del Coyote & 92 & 1010225 & 200658 & 1,970 & barberi \\
\hline Yuriria & 46 & Tejocote de Pastores & 87 & 1010338 & 200650 & 2,010 & barberi \\
\hline Yuriria & 46 & Timbinal & 91 & 1010250 & 200535 & 2,030 & barberi \\
\hline Yuriria & 46 & El Salteador & 60 & 1010436 & 200820 & 2,040 & barberi \\
\hline Yuriria & 46 & Providencia de Calera & 49 & 1012325 & 201100 & 2,050 & barberi \\
\hline
\end{tabular}

Reference codes are from INEGI databases are included for each registry.

health budgets, conduct diagnostic analyses of Chagas disease burden. Many states now recognize their Chagas disease problems, and the need to develop costeffective and integrated surveillance and control strategies, and so are attempting to complete preliminary risk assessments.

The first collection of triatomines in the state of Guanajuato, in central Mexico, was reported in 1956 by Biagi (Biagi 1956), for Triatoma pallidipennis (Stål) from Acambaro, Acambaro county. The only other previous report for triatomines was of Triatoma barberi (Usinger) from Los Galvanes, San Miguel de Allende county (Salazar-Schettino et al. 1983). Two seroepidemiological studies have been conducted in the state: the first, in 1991, at San José de La Presa, Purísima del Rincón county, found $6.1 \%$ of 228 samples from open population positive for antibodies to T. cruzi (Juarez-Leyva, personal communication). More recently, in 2000, in 60 communities in 12 counties, a seroprevalence of $2.6 \%(n=1730)$ was found (LópezCárdenas, personal communication).

The current study represents a first effort to identify the principal domestic triatomine species across the entire state of Guanajuato. To provide a landscapelevel view, without limitations imposed by sampling and completeness of inventory for the state, we use ecological niche modeling via evolutionary computing approaches. We then stratify Chagas transmission risk based on vector species distributions.

\section{Materials and Methods}

Study Site: Guanajuato State. Guanajuato is located in central Mexico in the high plains, with a total land area of $30,461 \mathrm{~km}^{2}$, corresponding to $1.5 \%$ of the country's total land mass. The northern half of the state lies within the Central Mesa region; the southern half in the Transverse Volcanic Belt; and the northeast corner touches the Sierra Madre Oriental. The state has three principal climatic zones: semidry, temperate, and semihot, with an average of $800 \mathrm{~mm}$ of rainfall concentrated between June and September. The state holds 46 administrative counties, four major urban centers (León, Irapuato, Salamanca, and Celaya, INEGI 2000), and eight Sanitary Jurisdictions for the Primary Health Care System.

Population Sampling. Communities from 43 of the 46 counties in Guanajuato were visited repeatedly (at least one visit per year and three visits per year in $12 \%$ of the counties) between October 1998 and July 2002 for Chagas disease prevention activities. All communities visited by health promotion personnel $(\approx 37 \%$ of all state communities), mobile medical units (Sanitary Jurisdictions 2 and 8), or the infant nutrition program PROGRESA were included in the study's activities. Health promotion personnel and vector technicians conducted community meetings, in which the population was given information regarding the disease and its vectors, bug recognition, and was requested to collect bugs observed in homes. Samples collected by the community (with name of family, date, habitat, community, and county) were sent to the Sanitary Jurisdiction (SJ) headquarters, and transported for analysis to the State Public Health Laboratory (León). Owing to the state's dispersed population, vector personnel from the SJ2 had greater contact directly with small isolated rural communities, and therefore they were able to survey a greater proportion of communities in the counties of Xichú, Victoria, and Sta. Catarina.

Analysis of Bug Collections and Entomological Indices. Data for all samples received by the State Public Health Laboratory were registered in a database. Bugs were identified using a standard key (Lent and Wygodzinsky 1979). Samples received live were examined for T. cruzi by direct observation of fecal samples in a standard light microscope. Standard entomological indices, including dispersion indices 
(number of positive villages per total villages examined $\times 100$ ), were calculated for communities and counties.

Ecological Niche Modeling. Our use of ecological niche modeling, which followed on several previous tests and prototypes (Peterson 2001, Beard et al. 2002, Costa et al. 2002, Peterson et al. 2002), introduces a new feature to the technique: the use of multitemporal, remotely sensed environmental data sets as a surrogate for climatic data (Rogers 2000, Egbert et al. 2002, Hay et al. 2002, Rogers et al. 2002). This environmental data set permits fine-scale predictions across landscapes for which climatic monitoring stations are too sparse to permit development of finescale climate maps.

Preliminary ecological niche models were based on unique occurrence records for all species encountered in the state, by using all known existing collection records (i.e., not part of the Guanajuato state sampling program described above) from 1948 to 2002 for the area within $250 \mathrm{~km}$ of Guanajuato. Three members of the phyllosoma species group and one from the protracta complex had sufficient unique occurrence localities (>30 records) for analysis. All occurrence points were georeferenced to the nearest $0.1^{\prime}$ of latitude and longitude via reference to Mexican census information (www.conabio.gob.mx, www.inegi.gob.mx). The Guanajuato state health survey was reserved as an independent test and validation of model predictivity.

Ecological niches were modeled using the Genetic Algorithm for Rule-set Prediction (GARP) (Stockwell and Noble 1992, Stockwell 1999, Stockwell and Peters 1999), an evolutionary-computing software package available for public download. In general, the procedure focuses on modeling ecological niches (the conjunction of ecological conditions within which a species is able to maintain populations without immigration) (Grinnell 1917). Specifically, GARP relates ecological characteristics of known occurrence points to those of points randomly sampled from the rest of the study region, seeking to develop a series of decision rules that best summarize those factors associated with the species' presence (Peterson et al. 2002a). In GARP, input occurrence points are divided randomly and evenly into training and (intrinsic) testing data sets. GARP works in an iterative process of rule selection, evaluation, testing, and incorporation or rejection: a method is chosen from a set of possibilities (e.g., logistic regression, bioclimatic rules), applied to the training data, and a rule is developed or evolved. Predictive accuracy is then evaluated based on 1,250 points resampled from the intrinsic test data and 1,250 points sampled randomly from the study region as a whole. Rules may evolve by a number of means that mimic DNA evolution: point mutations, deletions, and crossing over. The change in predictive accuracy from one iteration to the next is used to evaluate whether a particular rule should be incorporated into the model, and the algorithm runs either 1000 iterations or until convergence.
We used four data layers summarizing aspects of topography (elevation, slope, aspect, and topographic index [tendency to pool water]) from the U.S. Geological Survey's Hydro-1K data set, and 12 data layers summarizing monthly composite normalized difference vegetation index values ("greenness index") drawn from the Advanced Very High Resolution Radiometer satellite. Both data sets have a native spatial resolution of $\approx 1 \mathrm{~km}$ and were resolved to the same $1-\mathrm{km}$ grid across the region of study. GARP's predictive abilities have been tested and proven under diverse circumstances (Peterson et al. 1999, 2001, 2002c; Peterson 2001; Anderson et al. 2002, 2003).

To take advantage of the random-walk nature of the GARP algorithm, we developed 100 replicate models of each species' ecological niche. We used the procedures described in Anderson et al. (2003) for choosing a "best subset" of the 100 models, based on optimal combinations of error statistics. The procedure is based on the observations that 1) models vary in quality, 2) variation among models involves an inverse relationship between error of omission (leaving out true distributional area) and commission (including areas not actually inhabited), and 3) best models (as judged by experts blind to error statistics) are clustered in a region of minimum omission of independent test points and moderate area predicted (an axis related directly to commission error). The relative position of the cloud of points relative to the two error axes provides an assessment of the relative accuracy of each model. To choose best subsets of models, we 1) eliminated all models that had omission error $>5 \%$ based on independent intrinsic test points, 2) calculated the median area predicted present among these zero-omission points, 3 ) identified the 10 models closest to the overall median area predicted, and 4) summed these best subsets models.

Projection of the rule-sets for these best subsets models onto geography provided distributional predictions for species' ranges. Model quality was tested and validated via the independent Guanajuato state triatomine survey data. $\chi^{2}$ tests were used to compare observed success in predicting distributions of test points with those expected under random models (proportional area predicted present provides an estimate of occurrence points correctly predicted were the prediction to be random with respect to the distribution of the test points). Predicted presence was defined as the area in which all best-subsets models agreed in prediction of presence.

Disease Prevalence and Burden. Based on the areas identified by the ecological niche models at different levels of model agreement, human settlements in the state were ranked from lowest (1) to highest (10) for vector transmission risk. Three strata were defined based on this ranking: $1-3$, low risk; 4-7, medium risk; and 8-10, high risk. Community, demographic, and housing information from the 2000 National Census (INEGI 2000) were combined with the risk statistics, and total population at risk, seropositive population, incidence, mortality, and disease burden were calculated by county based on an overall 
Table 2. Communities collecting triatomines in Guanajuato counties; stratification of at-risk communities based on species collected and altitude ranges for each species in the state

\begin{tabular}{|c|c|c|c|c|c|c|c|c|}
\hline \multirow{2}{*}{ County } & \multicolumn{4}{|c|}{ Triatomines present } & \multirow{2}{*}{$\begin{array}{l}\text { No. comm. } \\
\text { at-risk }\end{array}$} & \multirow{2}{*}{$\begin{array}{l}\text { Comm. } \\
\text { with health } \\
\text { promotion }\end{array}$} & \multirow{2}{*}{$\begin{array}{l}\text { Comm. } \\
\text { infested }\end{array}$} & \multirow{2}{*}{$\begin{array}{c}\text { Collect success } \\
(\%)\end{array}$} \\
\hline & $800-1,200$ masl & $1,200-1,700$ masl & $1,700-1,900$ masl & $1,900-2,270$ masl & & & & \\
\hline 1 & & & $1, p$ & & 301 & 204 & 2 & 1.0 \\
\hline 2 & & & $\mathrm{~b}$ & $\mathrm{~b}$ & 213 & 80 & 1 & 1.3 \\
\hline 3 & & & $\mathrm{~m}$ & $\mathrm{~m}$ & 471 & 161 & 4 & 2.5 \\
\hline 4 & & & $\mathrm{~b}$ & $\mathrm{~b}$ & 122 & 52 & 2 & 3.8 \\
\hline 5 & & $\mathrm{~b}$ & $\mathrm{~b}$ & $\mathrm{~b}$ & 142 & 57 & 2 & 3.5 \\
\hline 6 & $\mathrm{~m}$ & $\mathrm{~m}$ & $\mathrm{~m}$ & $\mathrm{~m}$ & 36 & 14 & 11 & 81.5 \\
\hline 7 & & & b & $\mathrm{b}$ & 263 & 113 & 2 & 1.8 \\
\hline 8 & & & $\mathrm{~b}, \mathrm{l}, \mathrm{p}$ & $\mathrm{b}$ & 304 & 67 & 26 & 38.9 \\
\hline 9 & & & b & $\mathrm{b}$ & 150 & 54 & 6 & 11.1 \\
\hline 10 & & $\mathrm{~b}$ & $\mathrm{~b}$ & $\mathrm{~b}$ & 10 & 6 & 1 & 17.6 \\
\hline 11 & & & $\mathrm{~b}$ & $\mathrm{~b}$ & 109 & 53 & & \\
\hline 12 & & & $1, \mathrm{p}$ & & 89 & 12 & 1 & 8.4 \\
\hline 13 & & & & $\mathrm{~m}, \mathrm{~b}$ & 74 & 35 & & \\
\hline 14 & & & $\mathrm{~m}, \mathrm{~b}$ & $\mathrm{~m}, \mathrm{~b}$ & 526 & 73 & 18 & 24.6 \\
\hline 15 & & & $\mathrm{~b}$ & $\mathrm{~b}$ & 129 & 13 & & \\
\hline 16 & & & $\mathrm{l}, \mathrm{p}$ & & 38 & 22 & 2 & 9.2 \\
\hline 17 & & & $\mathrm{~b}, \mathrm{l}, \mathrm{p}$ & $\mathrm{b}$ & 472 & 191 & 10 & 5.2 \\
\hline 18 & & $\mathrm{~b}$ & b & & 38 & 19 & 1 & 5.1 \\
\hline 19 & & & & $\mathrm{~b}$ & 136 & 48 & & \\
\hline 20 & $\mathrm{~m}$ & $\mathrm{~m}$ & $\mathrm{~m}, \mathrm{l}$ & $\mathrm{m}$ & 509 & 240 & 4 & 1.7 \\
\hline 21 & & & $\mathrm{~b}$ & $\mathrm{~b}$ & 20 & 9 & 1 & 10.8 \\
\hline 22 & & & & $\mathrm{~b}$ & 73 & 54 & & \\
\hline 23 & & & $\mathrm{~b}, \mathrm{l}$ & $\mathrm{b}$ & 482 & 184 & 12 & 6.5 \\
\hline 24 & & & $\mathrm{l}, \mathrm{p}$ & & 52 & 28 & 2 & 7.0 \\
\hline 25 & & & $\mathrm{~b}, \mathrm{l}, \mathrm{p}$ & & 106 & 46 & & \\
\hline 26 & & & 1 & & 257 & 70 & 1 & 1.4 \\
\hline 27 & & & $\mathrm{~b}$ & $\mathrm{~b}$ & 309 & 156 & 4 & 2.6 \\
\hline 28 & & & $\mathrm{~b}$ & $\mathrm{~b}$ & 75 & 47 & & \\
\hline 29 & & & & $\mathrm{~b}$ & 190 & 44 & & \\
\hline 30 & & $\mathrm{~b}$ & $\mathrm{~b}$ & $\mathrm{~b}$ & 307 & 87 & 3 & 3.5 \\
\hline 31 & & & $1, \mathrm{p}$ & & 192 & 86 & 1 & 1.2 \\
\hline 32 & & & & $\mathrm{~m}, \mathrm{~b}$ & 193 & 109 & & \\
\hline 33 & $\mathrm{~m}$ & $\mathrm{~m}, \mathrm{~b}$ & $\mathrm{~m}, \mathrm{~b}$ & $\mathrm{~m}, \mathrm{~b}$ & 440 & 143 & 22 & 15.4 \\
\hline 34 & & $\mathrm{~m}, \mathrm{~b}, \mathrm{~d}$ & $\mathrm{~m}, \mathrm{~b}$ & $\mathrm{~m}, \mathrm{~b}$ & 41 & 26 & 26 & 100.0 \\
\hline 35 & & & $\mathrm{~b}$ & $\mathrm{~b}$ & 155 & 60 & 4 & 6.6 \\
\hline 36 & & & $\mathrm{~b}$ & $\mathrm{~b}$ & 14 & 6 & & \\
\hline 37 & & & $\mathrm{~b}$ & $\mathrm{~b}$ & 385 & 72 & 3 & 4.2 \\
\hline 38 & & & $\mathrm{~b}$ & $\mathrm{~b}$ & 26 & 10 & & \\
\hline 39 & & & $\mathrm{~b}$ & $\mathrm{~b}$ & 76 & 50 & 2 & 4.0 \\
\hline 40 & & & $\mathrm{~m}, \mathrm{~b}$ & $\mathrm{~m}, \mathrm{~b}$ & 48 & 37 & 37 & 100.0 \\
\hline 41 & & $\mathrm{~b}$ & $\mathrm{~b}$ & $\mathrm{~b}$ & 32 & 15 & 4 & 26.6 \\
\hline 42 & & $\mathrm{~b}$ & $\mathrm{~b}, \mathrm{l}$ & $\mathrm{b}$ & 231 & 28 & 9 & 32.4 \\
\hline 43 & $\mathrm{~m}$ & $\mathrm{~m}, \mathrm{~b}$ & $\mathrm{~m}, \mathrm{~b}$ & $\mathrm{~m}, \mathrm{~b}$ & 158 & 47 & 47 & 100.0 \\
\hline 44 & & & $\mathrm{~b}$ & $\mathrm{~b}$ & 120 & 80 & & \\
\hline 45 & $\mathrm{~m}$ & $\mathrm{~m}, \mathrm{~b}$ & $\mathrm{~m}, \mathrm{~b}$ & $\mathrm{~m}, \mathrm{~b}$ & 76 & 22 & 22 & 100.0 \\
\hline 46 & & & $\mathrm{~b}$ & $\mathrm{~b}$ & 106 & 44 & 11 & 25.3 \\
\hline Total & & & & & 6284 & 3073 & 247 & \\
\hline
\end{tabular}

amsl, above mean sea level; comm, community; b, T. barberi; d, T. dimidiata; 1, T. longipennis; m, T. mexicana; p, T. pallidipennis.

seroprevalence of 2.6\% (López-Cárdenas, personal communication).

\section{Results}

Among the 43 counties surveyed, after nearly $4 \mathrm{yr}$ of Chagas vector promotion activities, 32 (74\%; 201 communities) reported collections of bugs, representing $3.9 \%$ of all communities potentially at risk (Table 1). In total, 2,522 triatomine specimens of five species were collected: T. barberi, T. pallidipennis, Triatoma longipennis (Usinger), Triatoma mexicana (Herrich-Schaeffer), and Triatoma dimidata (Latreille) (one adult only). In 14\% of communities contributing bugs, more than one species of triatomine was found. The species most commonly found sympatric were 1) T. barberi, T. longipennis, and T. pallidipennis (three communities, one county); 2) T. barberi and T. mexicana (14 communities, five counties), 3) T. longipennis and T. pallidipennis (four communities, four counties), and 4) T. barberi and T. longipennis (seven communities, four counties).

Health promotion activities, mobile medical units, and/or vector control activities of the primary healthcare system covered an average of $37 \%$ of communities in the 43 counties (Table 2). Using altitudinal range, proportion of communities included in health promo- 
Table 3. Dispersion of domestic triatomines in at-risk communities in Guanajuato, 1998-2002

\begin{tabular}{lcccc}
\hline \hline \multicolumn{1}{c}{ Species } & $\begin{array}{c}\text { At-risk } \\
\text { communities } \\
\text { with health } \\
\text { promotion } \\
(n)\end{array}$ & $\begin{array}{c}\text { Communities } \\
\text { positive } \\
\text { for bugs } \\
(n)\end{array}$ & $\begin{array}{c}\text { Counties } \\
\text { positive } \\
\text { for bugs } \\
(n)\end{array}$ & $\begin{array}{c}\text { Dispersion } \\
\text { index (\%) }\end{array}$ \\
\hline T. barberi & 2,192 & 95 & 26 & \\
T. longipennis & 1,177 & 13 & 6 & 1.1 \\
T. pallidipennis & 656 & 31 & 12 & 4.7 \\
T. mexicana & 907 & 171 & 9 & 18.9 \\
\hline
\end{tabular}

tion activities, and vector species detected to stratify at-risk communities, a broad range of collection success was observed in each county $(0.4-77.1 \%)$, either reflecting real variation in dispersion indices or differences in community participation from county to county. There was a general tendency for higher "potential" dispersion indices $(>10 \%)$ only where T. mexicana or T. barberi were present, in the counties of Atarjea, Santa Catarina, Tierra Blanca, Victoria, and Xichú. Using estimated numbers of communities at risk where promotion activities were conducted, dispersion indices for the four triatomine species were $4.3,1.1,4.7$, and $18.9 \%$ for $T$. barberi, $T$. longipennis, T. pallidipennis, and T. mexicana, respectively (Table 3).

Bug collection sites inside and outside houses are reported in Table 4. T. barberi inside houses was found equally in bedrooms (23\%) and walls (24\%) and proportionally few from floors or ceilings; in the peridomicile, a high proportion $(33 \%)$ of collections was from animal corrals. Although few collections of T. longipennis and T. pallidipennis were from the intradomicile, greatest proportions of specimens were found in bedrooms (36 and $55 \%$, respectively), with fewer from walls ( 4 and $27 \%$, respectively), and even fewer from floors or ceilings. T. mexicana was found predominately on walls $(56 \%)$, followed by bedrooms $(25 \%)$; in the peridomicile, only $2 \%$ of samples were collected from animal corrals.

Due to often lengthy transport of bugs to the State Laboratory, not all specimens arrived in adequate condition for fecal examinations. Only 1,207 (47.9\%) of the total 2,522 specimens collected were examined for presence of Trypanosoma cruzi (Table 5). Few specimens of T. longipennis and T. pallidipennis were collected, although 45.5 and $7.7 \%$, respectively, of these were positive. From a total of 163 T. barberi and 1,009 T. mexicana, 19.0 and $2.9 \%$, respectively, were positive for T. cruzi.

Only one adult specimen of T. dimidiata was collected over the $4 \mathrm{yr}$, which may indicate the transport of an isolated specimen rather than domestic infestation. In addition to domestic specimens, only T. longipennis and T. mexicana were collected along rural roads in sylvan areas surrounding communities.

Ecological Niche and Transmission Risk Areas. All triatomine occurrence data points within $250 \mathrm{~km}$ of Guanajuato from a database that includes all registries (published or from state health authorities, but not including the survey described herein) of triatomine collections in Mexico from 1948 to present were modeled, and niche models were developed for all five species occurring in the state, although sample sizes were insufficient for T. dimidiata. Overlaying the independent Guanajuato state health survey data, the correspondence between prediction and independent test data were extremely good and highly statistically significant for $T$. longipennis $\left(\chi^{2}=34.7, P=3.78 \times\right.$ $\left.10^{-9}\right)$, T. pallidipennis $\left(\chi^{2}=16.4, P=5.24 \times 10^{-5}\right)$, and T. mexicana $\left(\chi^{2}=63.2, P=1.86 \times 10^{-15}\right)$, and significant if less close for $T$. barberi $\left(\chi^{2}=6.3, P=\right.$ 0.012 ). The only occurrence of $T$. dimidiata detected in the Guanajuato state health data set was not predicted as habitable for the species based on the ecological niche models.

Finally, the Guanajuato state health data set and the national data set were combined, and ecological niche model prediction maps generated for the four species confirmed to infest domestic habitats in the state (Figs. 1-4). Overall, Guanajuato has 8978 communities with more than two houses registered; 8,589 of these communities are predicted to have some risk of infestation by triatomine vectors, and hence vector transmission of Chagas disease (Table 6). Of the 8,589 communities at risk, 8,383 have "high" risk for infestation by at least one triatomine species. The species representing the greatest overall risk in terms of number of communities potentially inhabited are T. barberi and T. mexicana.

Using the highest risk prediction score from any of the four principal triatomine species, we estimate that $80.5 \%$ or $3,755,375$ inhabitants of Guanajuato are at direct risk for vector transmission among the total population of 4,665,000 (Table 6 ) and that $99.2 \%$ of this population is at "high" risk. Based on this latter at-risk

Table 4. Triatomine domestic collection sites inside and outside houses

\begin{tabular}{|c|c|c|c|c|c|c|c|c|}
\hline \multirow{2}{*}{ Species } & \multicolumn{6}{|c|}{ Intradomicile } & \multicolumn{2}{|c|}{ Peridomicile } \\
\hline & Bedroom & Floor & Wall & Roof & Kitchen & Other & Animal corral & Patio/garden \\
\hline T. barberi & 29 & 4 & 30 & 4 & 7 & $50 *$ & 9 & 18 \\
\hline T. longipennis & 9 & 3 & 1 & 1 & 2 & 9 & 1 & 5 \\
\hline T. pallidipennis & 6 & 0 & 3 & 0 & 0 & 2 & 1 & 1 \\
\hline T. mexicana & 96 & 21 & 216 & 3 & 7 & $43^{*}$ & 1 & 62 \\
\hline
\end{tabular}

Other includes sites inside houses, e.g., corridors, bathrooms, and living room.

a 32 for $T$. barberi and 36 for T. mexicana were broadly registered as 'intradomicile.' 


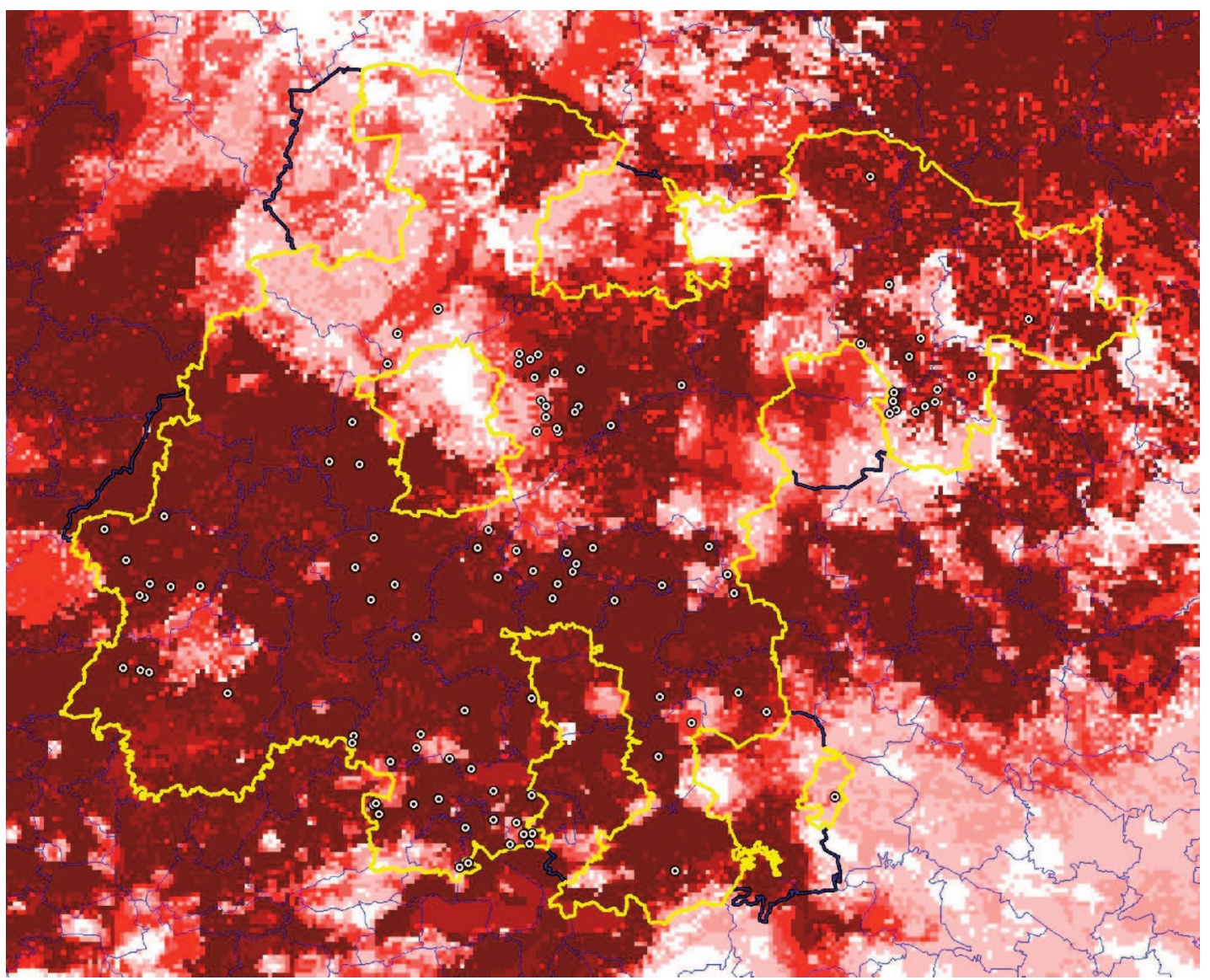

Fig. 1. Niche prediction area for T. barberi in the state of Guanajuato. Highest risk is indicated in dark red and lowest in white; collection sites are marked by open circles in the map.

population to calculate overall prevalence, and given a recent serological survey that found a seroprevalence of $2.6 \%$ for anti-T. cruzi antibody in the state, an estimated 97,640 inhabitants have Chagas disease in the state, of which 29,293 would currently be in the chronic phase (30\% of all cases). Incidence for Chagas cases is estimated as 3,485 per year, in the absence of any vector control measures. With 29,293 current chronic cases, we estimate that the state and/or the population spends an average U.S. $\$ 20,505,100$ in treatment alone, independent of whether the cases are recognized as Chagas, or as "cardiac insufficiency," where most of the current cases in Mexico are classified.

Table 5. Infection of triatomines with $T$. cruzi

\begin{tabular}{lccc}
\hline \multicolumn{1}{c}{ Species } & Examined & $\begin{array}{c}\text { Positive } \\
\text { T. cruzi }\end{array}$ & $\begin{array}{c}\text { T. cruzi } \\
\text { infection(\%) }\end{array}$ \\
\hline T. barberi & 163 & 31 & 19.0 \\
T. longipennis & 22 & 10 & 45.5 \\
T. pallidipennis & 13 & 1 & 7.7 \\
T. mexicana & 1009 & 29 & 2.9 \\
\hline
\end{tabular}

\section{Discussion}

Four triatomine vector species of Chagas disease have now been identified and their distributions modeled in the state of Guanajuato: T. barberi, T. longipennis, T. mexicana, and T. pallidipennis. All of these species are well adapted to domestic habitats, with colonization both inside and outside houses, and their T. cruzi transmission potential verified, with infection rates lowest for T. mexicana (6\%) and highest for T. longipennis (46\%). Despite collection bias, a result of more intensive health promotion and survey activities in T. mexicana-infested communities (highest collection success), this species has similar albeit slightly lower potential risk compared with $T$. barberi by using niche modeling. Both species are the most widely dispersed triatomines within the state and may infest all communities in certain counties. Neither T. longipennis nor T. pallidipennis were collected from $>10 \%$ of communities within given counties. These preliminary data will require verification through entomological surveys by using standardized methods and trained vector technicians. 


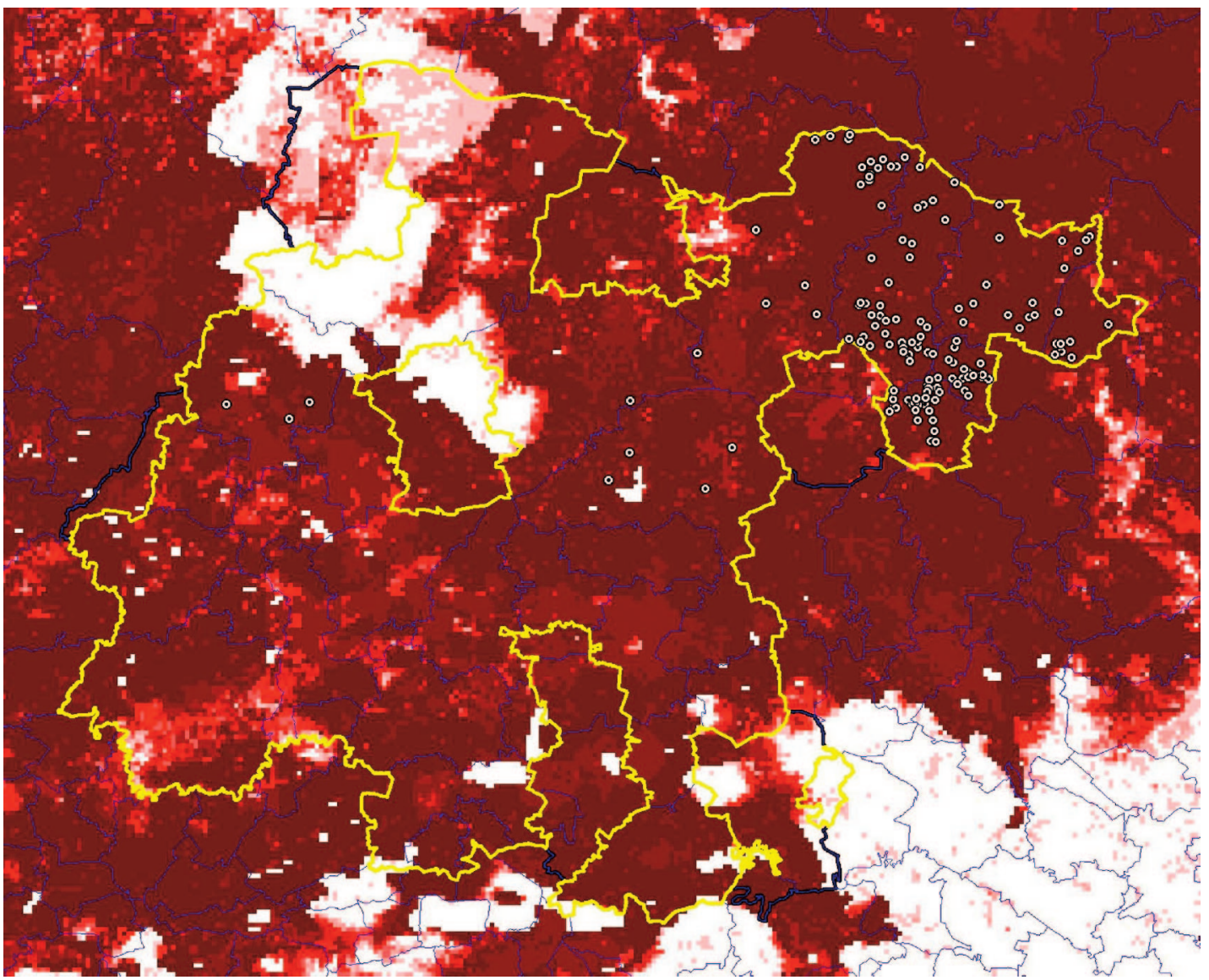

Fig. 2. Niche prediction area for T. mexicana in the state of Guanajuato. Highest risk is indicated in dark red and lowest in white; collection sites are marked by open circles in the map.

Most Mexican states are currently attempting to determine the distributions of their triatomine vectors to define at-risk populations for Chagas disease transmission (Ramsey et al. 2003) and to evaluate requirements for surveillance and control programs. This task is daunting, given the extent of the territory, and the lack of funds and personnel with which to conduct such surveys-indeed, it is clear that without local assistance and without more accessible methodology to measure vector distributions, diagnosis of the situation will remain obscure. This study succeeded in initiating a long-needed awareness program within the state for triatomines and Chagas disease, as well as intensive collection of vectors by local residents and vector-borne disease technicians. Nevertheless, it is clear that these collections must be supervised and supplemented with specific entomological surveys, at least in the initial stages of a surveillance program.

This study also presents a powerful example of the role that ecological niche modeling can play in this challenge (Peterson et al. 2002b). Niche-based predictions for species' distributions can be powerful tools with which health care services can outline vec- tor species' distributional areas, establish potential disease transmission risk areas, and determine real versus potential infestation. In our analysis, triatomine collection data from states within $250 \mathrm{~km}$ of Guanajuato coincided precisely with the local residents' collection data, and ecological niche models developed from the two data sets were virtually identical. In other words, using just the existing data from outside the state, we predicted the triatomine distributions with accuracy, thereby obviating the need for preliminary surveys. Such predictions could be used to design and guide on-ground survey efforts, thereby focusing effort considerably, and reducing costs significantly. These models can then be refined via secondary surveys conducted by trained technicians in sentinel counties, so that specific entomological indicators can be evaluated.

The case of the single individual of $T$. dimidiata collected is an important example of the potential use of ecological niche models to define risk. Using data sets from states surrounding Guanajuato, we conclude that this species has virtually no potential distributional area within the state. Collection of a lone adult 


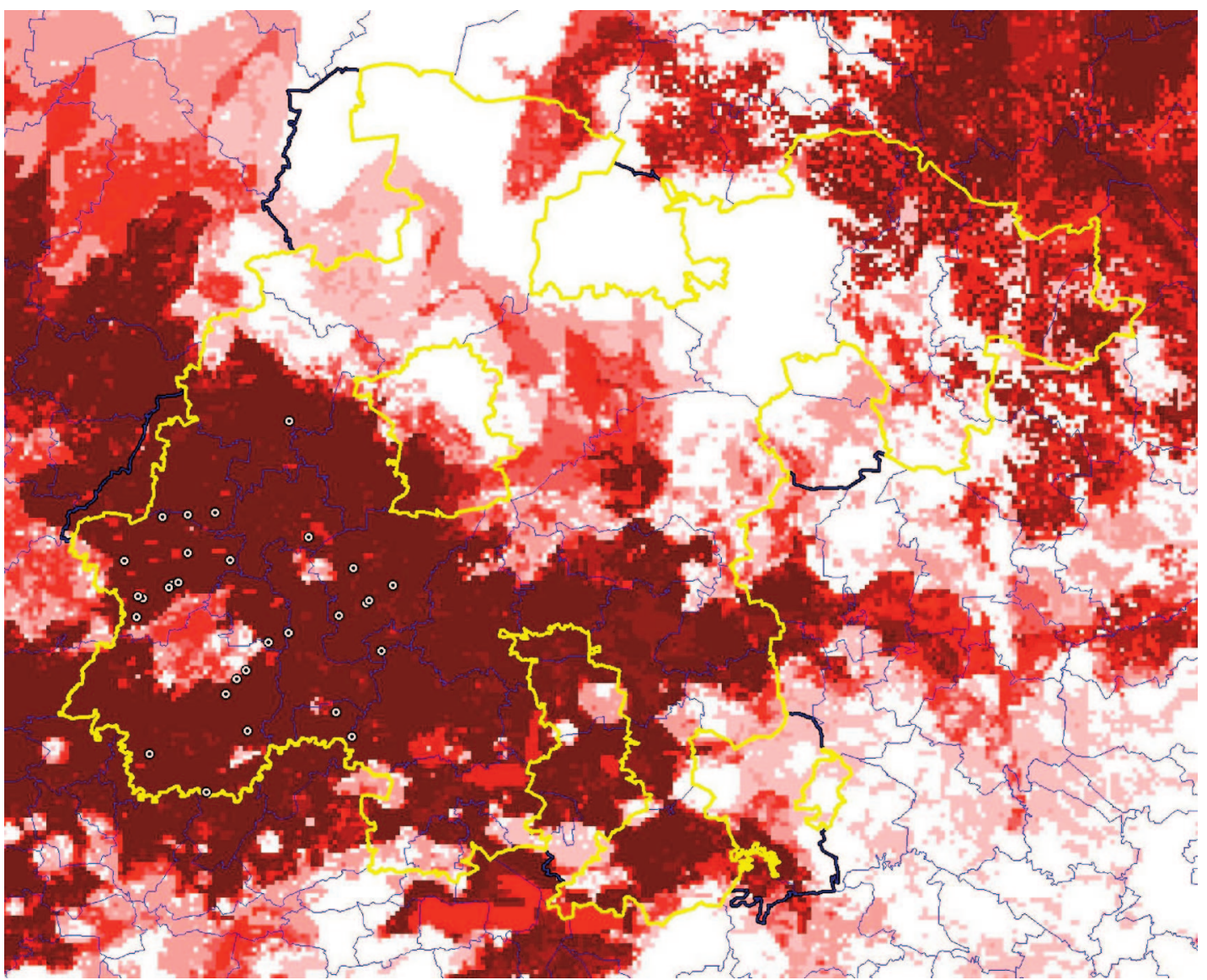

Fig. 3. Niche prediction area for T. longipennis in the state of Guanajuato. Highest risk is indicated in dark red and lowest in white; collection sites are marked by open circles in the map.

specimen can therefore be considered spurious, probably the result of human-assisted transport. Nevertheless, the lack of potential niche areas suggests that even though this species is transported periodically to the state, it probably cannot establish in sylvan habitats.

Chagas disease transmission risk is a function of the presence of triatomines in domestic habitats, which in turn is dependent on the presence of triatomines in surrounding sylvan areas. Niche modeling provides the only tool available to date for defining potential

Table 6. Predicted risk (high, score 15-20; medium, score 8-14; low, score 1-7) for vector transmission for all communities in Guanajuato by using GARP, and stratified by vector species

\begin{tabular}{lcccc}
\hline \hline \multicolumn{1}{c}{ Species } & $\operatorname{High}(\%)$ & Medium(\%) & Low $(\%)$ & Total \\
\hline T. barberi & $8,299(96.9)$ & $221(2.6)$ & $41(0.5)$ & 8,561 \\
T. longipennis & $5,132(72.9)$ & $601(8.5)$ & $1,307(18.6)$ & 7,040 \\
T. pallidipennis & $5,272(65.9)$ & $800(10.0)$ & $1,922(24.1)$ & 7,994 \\
T. mexicana & $7,504(88.9)$ & $497(5.9)$ & $436(5.2)$ & 8,437 \\
\hline
\end{tabular}

Note that sympatry and niche overlap among species results in repetition of at-risk communities. dispersion of individual triatomine species and hence potential transmission risk.

The use of prediction models for Chagas surveillance programs is currently feasible at both local and statewide levels. Control programs can use these models to determine at-risk populations, to monitor control program activities and to allow control strategies to be stratified according to real or potential risk (Ramsey et al. 2003). Potential infestation areas will require specific surveillance activities, different from actual infestation areas, and information processing from population-based collections or specific entomological surveys can feed the models so that they can accurately and rapidly track program needs. Most importantly, as demonstrated in this study, they can identify preliminary risk areas so that vector control technicians can direct their surveys cost-effectively for more precise and local infestation indicators.

\section{Acknowledgments}

We thank the invaluable collaboration of all personnel from the primary health care services in the state of Guana- 


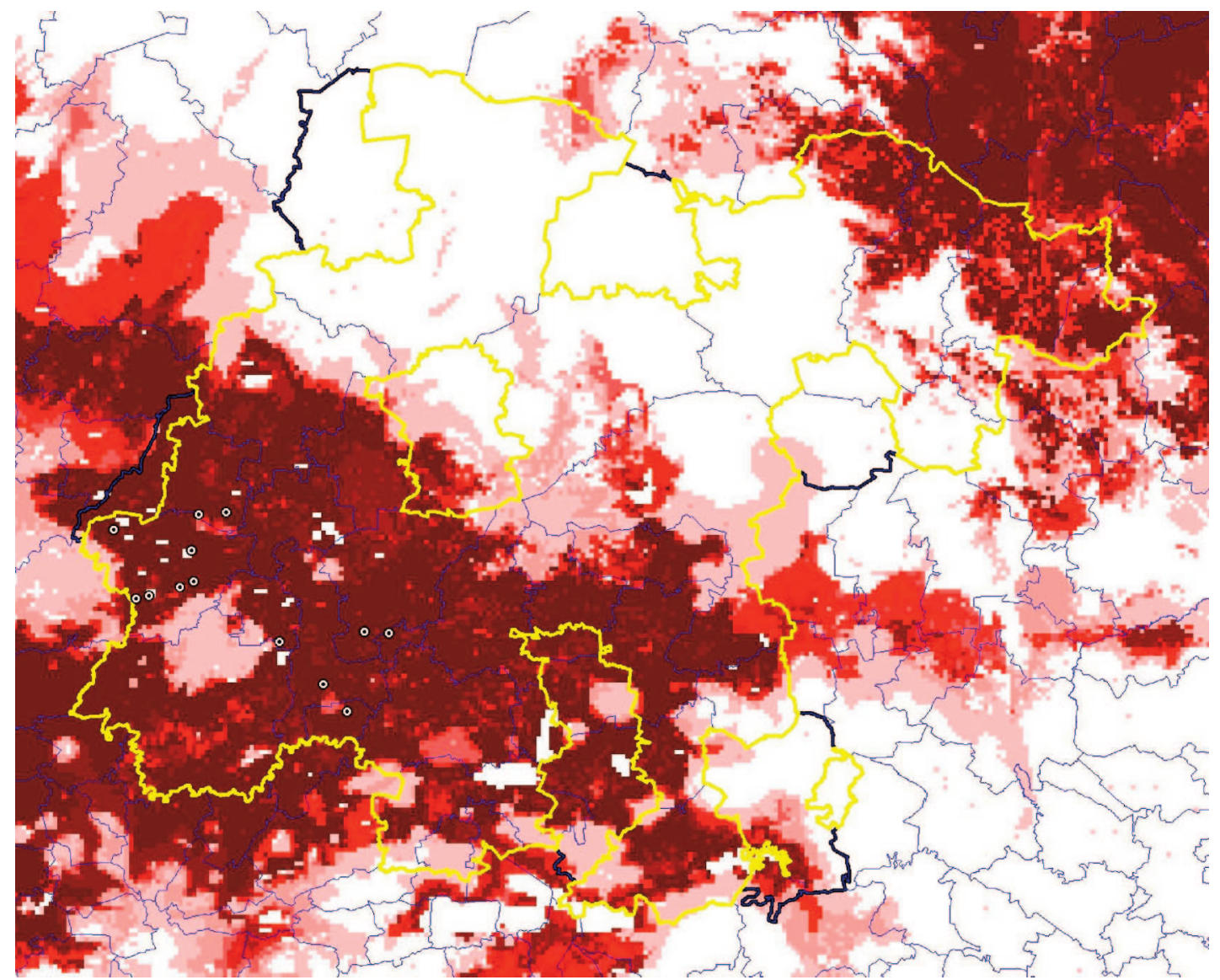

Fig. 4. Niche prediction area for T. pallidipennis in the state of Guanajuato. Highest risk is indicated in dark red and lowest in white; collection sites are marked by open circles in the map.

juato, especially the state and Sanitary Jurisdiction epidemiologists and technical personnel from the vector control program. We thank Vianey Vidal from the National Laboratory for Diagnosis and Epidemiological Reference for assistance with quality control of taxonomic identifications and Gely Peña Arguntia for dedication in health promotion and county collection activities. We also thank the Vector Control Coordinators from the states of Mexico, Morelos, San Luis Potosi, and Veracruz for provision of data regarding Triatoma distributions not reported in the scientific literature and to the Comisión Nacional para la Conservación y Uso de la Biodiverisdad (CONABIO) for access to mammal distributional data. This study was financed through the Guanajuato State Health budget, federal project funds from the National Institute of Public Health Mexico to J.M.R., and by the U.S. National Science Foundation and Consejo Nacional para Ciencia y Tecnología to A.T.P.

\section{References Cited}

Anderson, R. P., M. Laverde, and A. T. Peterson. 2002. Geographical distributions of spiny pocket mice in South America: insights from predictive models. Global Ecol. Biogeogr 11: 131-141.

Anderson, R. P., D. Lew, and A. T. Peterson. 2003. Using intermodel variation in error components to select best subsets of ecological niche models. Ecol. Model. 162: 211-232.
Beard, C. B., G. Pye, F. J. Steurer, Y. Salinas, R. Campman, A. T. Peterson, J. M. Ramsey, R. A. Wirtz, and L. E. Robinson. 2002. Chagas disease in a domestic transmission cycle in southern Texas, USA. Emerg. Infect. Dis. 9: $103-105$.

Biagi, F. 1956. Nueva Infección natural con Schizotrypanum cruzi. Variabilidad cromática y otras notas sobre triatomas Mexicanas. Prensa Med. Mex 21: 123-126.

Costa, J., A. T. Peterson, and C. B. Beard. 2002. Ecological niche modeling and differentiation of populations of Triatoma brasiliensis Neiva, 1911, the most important Chagas disease vector in northeastern Brazil (Hemiptera, Reduviidae, Triatominae). Am. J. Trop. Med. Hyg. 67: 516-520.

Egbert, S. L., E. Martinez-Meyer, M. A. Ortega-Herta, and A. T. Peterson. 2002. Use of datasets derived from timeseries AVHRR imagery as surrogates for land cover maps in predicting species' distributions. Proceedings IEEE 2002. Int. Geo. Rem. Sen. Symp. (IGARSS) 4: 2337-2339.

Grinnell, J. 1917. Field tests of theories concerning distributional control. Am. Nat. 51: 115-128.

Hay, S. I., M. F. Myers, N. Maynard, and D. J. Rogers. 2002. From remote sensing to relevant sensing in human health. Photogrammetric Eng. Remote Sensing 68: 109-111.

Lent, H., and P. Wygodzinsky. 1979. Revision of the Triatominae (Hemiptera: Reduviidae), and their significance as vectors of Chagas disease. Bull. Amer. Mus. Nat. Hist. 163: 520 . 
López-Cárdenas, J. 2001. Identificación y distribución espacial de las especies de vectores de la enfermedad de Chagas en el Estado de Guanajuato, de 1998-2000. M.S. thesis, Universidad de Guanajuato, León, Guanajuato, México.

Peterson, A. T. 2001. Predicting species' geographic distributions based on ecological niche modeling. Condor 103: 599-605.

Peterson, A. T., and D. A. Vieglais. 2001. Predicting species invasions using ecological niche modeling. BioScience 51: 363-371.

Peterson, A. T., J. Soberon, and V. Sanchez-Cordero. 1999. Conservatism of ecological niches in evolutionary time. Science (Wash. DC) 285: 1265-1267.

Peterson, A. T., V. Sanchez-Cordero, J. Soberon, J. Bartley, R. H. Buddemeier, and A. G. Navarro-Siguenza. 2001. Effects of global climate change on geographic distributions of Mexican Cracidae. Ecol. Model. 144: 21-30.

Peterson, A. T., M. A. Ortega-Huerta, J. Bartley, V. SanchezCordero, J. Soberon, R. H. Buddemeier, and D.R.B. Stockwell. 2002a. Future projections for Mexican faunas under global climate change scenarios. Nature (Lond.) 416: 626-629.

Peterson, A. T., V. Sanchez-Cordero, C. B. Beard, and J. M. Ramsey. 2002b. Ecologic niche modeling and potential reservoirs for Chagas disease, Mexico. Emerg. Infect. Dis. 8: 662-667.

Peterson, A. T., D.R.B. Stockwell, and D. A. Kluza. 2002c. Distributional prediction based on ecological niche modeling of primary occurrence data, pp. 617-623. In J. M. Scott [ed.], Predicting species occurrences: Issues of scale and accuracy. Island Press, Washington, DC.

Peterson, A. T., D. A. Vieglais, and J. Andreasen. 2005. .Migratory birds as critical transport vectors for West Nile Virus in North America. Emerg. Inf. Dis. (in press).

Ramsey, J. M., R. Ordoñez, A. Tello Lopez, J. L. Pohls, V. Sanchez-Cordero, and A. T. Peterson. 2003. Actuali- dades sobre la epidemiología de la enfermedad de Chagas en México, pp. 85-105. In J. M. Ramsey, A. Tello Lopez, and J. L. Pohls [eds.], Iniciativa para la vigilancia y control de la enfermedad de Chagas en México. Instituto Nacional de Salud Pública, México.

Rogers, D. J. 2000. Satellites, space, time and the African trypanosomiases, pp. 130-171. In S. I. Hay, S. Randolph, and D. J. Rogers [eds.], Remote sensing and GIS in public health. Academic, New York.

Rogers, D. J., S. E. Randolph, R. W. Snow, and S. I. Hay. 2002. Satellite imagery in the study and forecast of malaria. Nature (Lond.) 415: 710-715.

Salazar-Schettino, P. M., I de Haro, J. Jiménez, and E. Garcia Carrera. 1983. Dos nuevas localidades de transmisores de la enfermedad de Chagras en la República Mexicana. Salud Pública Méx. 25: 77-82.

Stockwell, D.R.B. 1999. Genetic algorithms II, pp. 123-144. In A. H. Fielding [ed.], Machine learning methods for ecological applications. Kluwer Academic Publishers, Boston, MA.

Stockwell, D.R.B., and I. R. Noble. 1992. Induction of sets of rules from animal distribution data: a robust and informative method of analysis. Math. Comp. Simul. 33: 385390.

Stockwell, D.R.B., and D. P. Peters. 1999. The GARP modelling system: problems and solutions to automated spatial prediction. Int. J. Geogr. Inf. Sys. 13: 143-158.

Vidal-Acosta, V., S. Ibáñez-Bernal, and C. Martínez-Campos. 2000. Infección Natural de chinches Triatominae con Trypanosoma cruzi asociadas a la vivienda humana en México. Salud Pública de Mex. 42: 496-503.

Zárate, L. G., and R. J. Zárate. 1985. A checklist of the Triatominae (Hemiptera: Reduvidae). Int. J. Entomol. 27: $102-127$.

Received 19 March 2004; accepted 25 January 2005. 